Meta

Journal des traducteurs

Translators' Journal

\title{
Rectifier le tir sur la triade faux sens, contresens, non-sens
}

\section{Éric Poirier}

Volume 60, numéro 2, août 2015

$60^{\mathrm{e}}$ anniversaire. Les horizons de la traduction : retour vers le futur

$60^{\text {th }}$ Anniversary. Translation's Horizons: Back to the Future

$60 \mathrm{mo}$ aniversario. Los horizontes de la traducción: regreso al futuro

URI : https://id.erudit.org/iderudit/1032915ar

DOI : https://doi.org/10.7202/1032915ar

Aller au sommaire du numéro

Éditeur(s)

Les Presses de l’Université de Montréal

ISSN

0026-0452 (imprimé)

1492-1421 (numérique)

Découvrir la revue

Citer ce document

Poirier, É. (2015). Rectifier le tir sur la triade faux sens, contresens, non-sens. Meta, 60(2), 364-364. https://doi.org/10.7202/1032915ar d'utilisation que vous pouvez consulter en ligne.

https://apropos.erudit.org/fr/usagers/politique-dutilisation/ 


\title{
Rectifier le tir sur la triade faux sens, contresens, non-sens
}

\author{
Éric Poirier \\ Université du Québec à Trois-Rivières, Trois-Rivières, Canada \\ Eric.Poirier@uqtr.ca
}

Les difficultés d'application de la triade du faux sens, du contresens et du non-sens semblent avoir eu raison de ces concepts hérités de la traduction universitaire francophone. Comme l'a proposé Dussart (2005), dans Meta (il y a tout juste 10 ans), la triade a totalement disparu des schémas d'évaluation des traductions. Elle a disparu aussi des contenus pédagogiques de la plupart des manuels de traduction francophones comme ceux de Jean Delisle et Marco Fiola (2013), de Daniel Gile (2005), de Valentine et Aubin (2004), de Claude Bédard (1986), en traduction technique, de Federica Scarpa (2010), en traduction spécialisée. Et l'on n'en trouve aucune trace dans les concepts clés d'une réflexion théorique comme celle de Mathieu Guidère (2010).

Malgré les progrès accomplis depuis 60 ans, il reste encore beaucoup d'incertitudes et même une part de mystère concernant le traitement du sens dans l'opération de la traduction. Étant donné le rôle prépondérant du sens en traduction, la traductologie peut difficilement faire l'économie de ces concepts fondamentaux dans la description de l'opération de la traduction, et surtout dans l'apprentissage empirique ou par essaierreur du transfert du sens qui s'appuie sur l'évitement des erreurs ou des écarts de sens dans l'opération traduisante (Collombat 2009). Sur le plan pédagogique, le faux sens, le contresens et le non-sens offrent aussi l'avantage de proposer une gradation utile dans l'évaluation des erreurs de transfert. Encore faut-il comme l'a bien vu Dussart que ces concepts puissent être dotés d'une signification différenciée, ce qui permettrait d'établir des critères précis (et indiscutables) de définition de ces erreurs de transfert du sens.

C'est ce que nous souhaitons proposer en présentant les critères définitionnels nécessaires à la différenciation des faux sens, des contresens et des non-sens. Ces propositions découlent en bonne partie des résultats obtenus dans des activités d'apprentissage sur le repérage de faux sens, contresens et non-sens qui ont été conçues pour le cours de méthodologie de la traduction donné en ligne à l'UQTR depuis l'automne 2012.

Éric Poirier, traducteur agréé, est professeur adjoint à l'Université du Québec à Trois-Rivières où il conçoit et donne des cours en ligne de traduction générale et spécialisée de l'anglais au français dans des programmes de formation professionnelle de premier cycle entièrement offerts en ligne. Il détient un doctorat en traduction avec spécialisation en lexicographie bilingue de l'Université de Montréal et il a exercé le métier de traducteur professionnel dans de grandes entreprises, en cabinet et à son compte pendant près de 20 ans. Il est l'auteur du Bestiaire, un dictionnaire de traduction analogique des expressions animalières de la langue anglaise. Ses publications récentes portent sur la linguistique de corpus en traductologie, le repérage des unités phraséologiques, les ruptures lexicogrammaticales en traduction et les technologies du traitement des langues naturelles dans l'instrumentation de l'enseignement de la traduction. 